\title{
Concentrações de FSH, LH, estradiol, progesterona e histamina no soro, no fluido peritoneal e no fluido folicular de mulheres com e sem endometriose
}

\author{
FSH, LH, estradiol, progesterone, and histamine concentrations in serum, \\ peritoneal fluid and follicular fluid of women with and without endometriosis
}

Andréa Pereira de Lima $^{1}$, Alzira Amélia Martins Rosa e Silva ${ }^{2}$, Marcos Dias de Moura ${ }^{3}$

\section{Resumo}

\begin{abstract}
Objetivos: relatos da literatura mostram que não há dados conclusivos sobre a associação entre a endometriose e as concentrações de hormônios envolvidos no controle da reprodução. Este estudo foi realizado para determinar as concentrações de FSH, LH, estradiol (E), progesterona (P) e histamina (Hi) no soro, no fluido peritoneal e no fluido folicular de mulheres com e sem endometriose. Métodos: a extensão da doença foi estadiada de acordo com a American Fertility Society classification (1997). Para a coleta de soro e de fluido peritoneal foram selecionadas 28 mulheres com endometriose submetidas à laparoscopia diagnóstica (18 mulheres inférteis com endometriose I-II e dez mulheres inférteis com endometriose IIIIV). Para o grupo controle, foram selecionadas 21 mulheres férteis submetidas à laparoscopia para esterilização tubárea. O fluido folicular foi obtido de 39 mulheres inférteis submetidas a fertilização in vitro (21 mulheres com endometriose e 18 mulheres sem endometriose). Resultados: as concentrações de FSH e LH no soro, no fluido peritoneal e no fluido folicular não diferiram significativamente entre os grupos. As concentrações de E e P no fluido peritoneal foram significativamente mais baixas em mulheres inférteis com endometriose (E: $154,2 \pm 15,3$ para estágios I-II e $89,3 \pm 9,8 \mathrm{ng} / \mathrm{mL}$ para

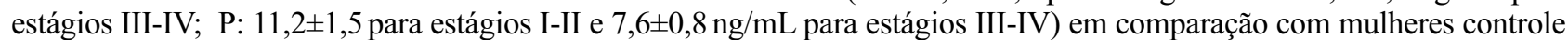
(E: 289,1 $\pm 30,1 ; P: 32,8 \pm 4,1$ ng/mL) (Testes de Kruskal-Wallis/Dunn; p<0,05). No soro, as concentrações de E e P seguiram o mesmo padrão. No fluido folicular, as concentrações de $\mathrm{E}$ e Hi foram significativamente mais baixas em mulheres com endometriose (E: $97,4 \pm 11,1 \mathrm{ng} / \mathrm{mL}$; Hi: $6,6 \pm 0,9 \mathrm{ng} / \mathrm{mL})$ em comparação com mulheres sem endometriose (E: 237,5 $\pm 28,5$ pg/mL; Hi: $13,8 \pm 1,3 \mathrm{ng} / \mathrm{mL}$ ), enquanto os níveis de P não revelaram diferença significativa entre os grupos (teste $t$ de Student; $\mathrm{p}<0,05$ ). Conclusões: nossos resultados indicam disfunção ovariana em mulheres com endometriose, com redução nas concentrações de estradiol, progesterona e histamina, o que pode contribuir para a subfertilidade freqüentemente associada à doença.
\end{abstract}

PALAVRAS-CHAVE: Hormônio folículo estimulante; Hormônio luteinizante; Progesterona; Estradiol; Histamina; Endometriose; Infertilidade feminina; Fertilização in vitro

\section{Abstract}

Purpose: literature reports show that there are no conclusive data about the association between endometriosis and the concentrations of hormones involved in the control of reproduction. Thus, the present study was undertaken to determine FSH, LH, estradiol (E), progesterone (P), and histamine (Hi) concentrations in serum, peritoneal fluid and follicular fluid of women with and without endometriosis. Methods: the extent of the disease was staged according to the revised American Fertility Society classification (1997). For the collection of serum and peritoneal fluid, 28 women with endometriosis undergoing diagnostic laparoscopy were selected (18 infertile women with endometriosis I-II and ten infertile women with endometriosis III-IV). For the control group, 21 fertile women undergoing laparoscopy for tubal sterilization were selected. Follicular fluid was obtained from 39 infertile women undergoing in vitro fertilization (21 women with endometriosis and 18 women without endometriosis). Results: FSH and LH levels in serum, peritoneal fluid and follicular fluid did not differ significantly between groups. On the other hand, E and P concentrations in the peritoneal fluid were significantly lower in infertile women with endometriosis (E: $154.2 \pm 15.3$ for stages I-II and $89.3 \mathrm{ng} / \mathrm{mL} \pm 9.8 \mathrm{ng} / \mathrm{mL}$ for stages III-IV; P: $11.2 \pm 1.5$ for stages I-II and $7.6 \mathrm{ng} / \mathrm{mL} \pm 0.8$ for stages III-IV) in comparison with control women $(\mathrm{E}: 289.1 \mathrm{ng} / \mathrm{mL} \pm 30.1$; P: $32.8 \pm 4.1 \mathrm{ng} / \mathrm{mL}$ ) (Kruskal-Wallis/Dunn tests; $\mathrm{p}<0.05$ ). In serum, estradiol and progesterone concentrations followed

Trabalho realizado na Clínica Ginecológica do Departamento de Ginecologia e Obstetrícia do Hospital das Clinicas da Faculdade de Medicina de Ribeirão Preto da Universidade de São Paulo - USP - Ribeirão Preto (SP) - Brasil.

Apoio: Fundação de Amparo à Pesquisa do Estado de São Paulo - FAPESP - São Paulo (SP), Brasil.

1 Pós-graduanda do Departamento de Fisiologia da Faculdade de Medicina da Universidade de São Paulo - USP - Ribeirão Preto (SP) - Brasil.

2 Docente do Departamento de Fisiologia da Faculdade de Medicina da Universidade de São Paulo - USP - Ribeirão Preto (SP) - Brasil.

3 Docente do Departamento de Ginecologia e Obstetrícia do Hospital das Clínicas da Faculdade de Medicina da Universidade de São Paulo - USP - Ribeirão Preto (SP) - Brasil.

Correspondência: Andréa Pereira de Lima

Rua Dr. Sirley Pereira Arantes 73, apto. 204 - Bairro Finotti - 38408735 - Uberlândia - MG - Telefone: (34) $3218-2200$ / (34) 3224-1402 e-mail: apljh@hotmail.com 
the same pattern. In the follicular fluid, E and Hi concentrations were significantly lower in women with endometriosis (E: $97.4 \pm 11.1 \mathrm{pg} / \mathrm{mL}$; Hi: $6.6 \pm 0.9 \mathrm{ng} / \mathrm{mL}$ ) in comparison to women without endometriosis (E: $237.5 \pm 28.5 \mathrm{pg} / \mathrm{mL}$; Hi: $13.8 \pm 1.3 \mathrm{ng} / \mathrm{mL}$ ) (Student $t$-test; $\mathrm{p}<0.05$ ), while progesterone levels revealed no significant difference between groups. Conclusions: our results indicate ovary dysfunction in women with endometriosis, with reduction on $\mathrm{E}, \mathrm{P}$ and $\mathrm{Hi}$ concentrations, which may contribute to the subfertility often associated with the disease.

KEYWORDS: Follicle stimulating hormone; Luteinizing hormone; Progesterone; Estradiol; Histamine; Endometriosis; Infertility, female; Fertilization in vitro

\section{Introdução}

A endometriose é definida como a presença de tecido endometrial fora do útero ${ }^{1}$. O tecido endometriótico implanta-se durante a menstruação, principalmente em locais como o peritôneo e os ovários, e desenvolve um suprimento vascular eficiente, o qual permite seu posterior crescimento no local de implantação ${ }^{2}$. O desenvolvimento de tecido endometriótico fora do útero parece estar associado a profundas alterações do sistema imunológico em mulheres com endometriose. A menstruação retrógrada, fenômeno comum em mulheres em idade reprodutiva, poderia facilitar o implante de células endometrióticas na cavidade peritoneal, embora nem todas as mulheres com menstruação retrógrada apresentem endometriose ${ }^{3}$.

A endometriose pode ser considerada como um processo inflamatório pélvico, com função alterada de células do sistema imunológico e número aumentado de macrófagos ativados no fluido peritoneal, os quais secretam vários fatores locais, tais como fatores de crescimento e citocinas ${ }^{4}$. Assim, o desenvolvimento da endometriose parece ser um fenômeno complexo, facilitado por fatores múltiplos, incluindo quantidade e qualidade de células endometriais no fluido peritoneal, atividade inflamatória aumentada no fluido peritoneal, expressão aumentada de moléculas de adesão, angiogênese aumentada e eliminação reduzida das células endometrióticas por um sistema imunológico deficiente ${ }^{3}$.

Esta doença complexa é um problema ginecológico comum e, freqüentemente, associado à infertilidade e dor pélvica ${ }^{1}$. A deficiência reprodutiva associada à endometriose e à infertilidade idiopática estão ainda entre os problemas mais dificeis que o ginecologista enfrenta. Um número significativo de mulheres que se queixam de dismenorréia e dor pélvica têm endometriose e mais da metade das mulheres com infertilidade não explicada são diagnosticadas com endometriose por laparoscopia 5 .

Embora a associação entre a endometriose e a infertilidade (ou estritamente subfertilidade) seja mostrada por estudos de prevalência, a causalidade ainda não está bem definida. A endometriose é associada com alterações no eixo hipotálamo-hipófise-ovário, com conseqüentes alterações nas concentrações do hormônio foliculo-estimulante (FSH), do hormônio luteinizante (LH), de estradiol e progesterona, no soro, no fluido peritoneal e no fluido folicular de mulheres com endometriose. A associação da endometriose com alterações no eixo hipotálamohipófise-ovário sugere uma desordem na função folicular, com concentração alterada do hormônio luteinizante, capacidade reduzida do oócito para fertilização e função lútea alterada ${ }^{6,7}$.

Estudos anteriores em mulheres com endometriose têm demonstrado: crescimento folicular prejudicado, redução nas concentrações plasmáticas de estradiol durante a fase pré-ovulatória e redução de estradiol e progesterona durante a fase lútea, além de padrões alterados de surto de LH. Uma redução nas concentrações de LH no fluido folicular também já foi relatada, e células granulosas coletadas no mesmo período podem apresentar capacidade esteroidogênica prejudicada $^{6,7}$.

Uma análise crítica dos dados da literatura mostra que não há dados conclusivos sobre a associação entre as concentrações de fatores envolvidos na reprodução e endometriose. Além disso, segundo nosso conhecimento, não há relato na litaratura sobre as concentrações de histamina no fluido peritoneal e no fluido folicular em mulheres com endometriose, embora tenha sido sugerido que a histamina também é importante para uma ovulação normal e implantação do blastocisto, regulação do fluxo sanguíneo na placenta, lactação e contratilidade uterina ${ }^{8-10}$.

Uma vez que mulheres com endometriose apresentam anormalidades reprodutivas, tais como disfunção ovulatória e foliculogênese alterada, o presente estudo foi realizado para determinar as concentrações de FSH, LH, estradiol, progesterona e histamina, no soro, no fluido peritoneal e no fluido folicular de mulheres com e sem endometriose. 
Métodos

Foram selecionadas mulheres atendidas nos ambulatórios da Clínica Ginecológica do Departamento de Ginecologia e Obstetrícia do Hospital das Clínicas da Faculdade de Medicina de Ribeirão Preto, da Universidade de São Paulo, USP (casos consecutivos). O período de desenvolvimento do estudo foi de 2002 a 2004.

Este trabalho foi aprovado pelo Comitê de Ética do Hospital das Clínicas da Faculdade de Medicina de Ribeirão Preto. Todas as pacientes incluídas neste estudo foram informadas dos procedimentos a serem realizados para a obtenção do material de pesquisa, conforme orientação da Comissão de Normas Éticas e Regulamentares da Faculdade de Medicina de Ribeirão Preto da USP.

A extensão da endometriose foi estadiada de acordo com os critérios da American Society for Reproductive Medicine (ASRM) ${ }^{11}$. O sistema de classificação da ASRM baseia-se no aspecto, tamanho e profundidade de implantes peritoneais e ovarianos; na presença, extensão e tipo de aderências; e no grau de obliteração do fundo de saco. Estes parâmetros, em conjunto, refletem a extensão da doença endometriótica. Os estádios são dependentes da pontuação de acordo com o indicado abaixo:

- Estágio I (endometriose mínima): escore 1-5

- Estágio II (endometriose leve): 6-15

- Estágio III (endometriose moderada): 16-40

- Estágio IV (endometriose grave): $>40$

Para a coleta de soro e de fluido peritoneal foram selecionadas 49 pacientes, sendo 21 mulheres férteis sem endometriose submetidas à laparoscopia para esterilização tubárea e 28 mulheres inférteis com endometriose pélvica, submetidas à laparoscopia diagnóstica por queixa de infertilidade e/ou dor pélvica. Entre as 28 mulheres inférteis com endometriose pélvica, 18 mulheres tinham endometriose nos estágios I-II e dez mulheres tinham endometriose nos estágios III-IV.

Para a coleta de fluido folicular foram selecionadas 39 pacientes inférteis submetidas à fertilização in vitro, sendo 18 mulheres inférteis sem endometriose e 21 mulheres inférteis com endometriose. Ambos os grupos de pacientes fizeram tratamento para indução de superovulação que precedeu a coleta dos oócitos, e, portanto, do fluido folicular.

A infertilidade foi definida como a incapacidade de conseguir gravidez após o mínimo de um ano de intercurso, sem o uso de métodos contraceptivos.
Para o grupo de mulheres férteis sem endometriose, os critérios de inclusão e exclusão para a coleta de soro e de fluido peritoneal foram: coleta realizada na fase secretora do ciclo menstrual; ausência de endometriose no momento da laparoscopia; ausência de qualquer tratamento clínico prévio à laparoscopia no período de, no minimo, três meses; ausência de outras doenças ginecológicas; ausência de dor pélvica na forma de dismenorréia ou algia pélvica crônica; presença de fluido peritoneal na cavidade abdominal durante a laparoscopia e idade superior a 18 anos e inferior a 40 anos.

No grupo de mulheres inférteis com endometriose, os critérios para coleta de soro e fluido peritoneal foram: coleta realizada na fase secretora do ciclo menstrual; diagnóstico de endometriose comprovado por laparoscopia; ausência de qualquer tratamento clínico prévio à laparoscopia no período de, no mínimo, três meses; ausência de outras patologias clínicas ou ginecológicas associadas à endometriose; presença de fluido peritoneal na cavidade abdominal durante a laparoscopia e idade superior a 18 anos e inferior a 40 anos.

Os critérios de inclusão e exclusão para a coleta de fluido folicular no grupo das mulheres inférteis sem endometriose submetidas à fertilização in vitro foram: ausência de endometriose no momento da laparoscopia; ausência de outras patologias clínicas ou ginecológicas associadas à endometriose; ausência de dor pélvica na forma de dismenorréia ou algia pélvica crônica e idade superior a 18 anos e inferior a 40 anos.

O grupo das mulheres inférteis com endometriose submetidas à fertilização in vitro tinha como caracaterísticas: diagnóstico de endometriose comprovado por laparoscopia (o intervalo entre o diagnóstico da endometriose e o início do tratamento para a fertilização in vitro variou de um a seis meses); ausência de outras patologias clínicas ou ginecológicas associadas à endometriose e idade superior a 18 anos e inferior a 40 anos.

\section{Coleta de fluido peritoneal}

As amostras de fluido peritoneal foram obtidas durante a laparoscopia realizada na fase secretora do ciclo menstrual, indicada para avaliação de infertilidade, dor pélvica ou esterilização tubárea. O fluido peritoneal foi aspirado do espaço uterovesical anterior e do fundo de saco de Douglas, assim que a cavidade pélvica foi visualizada. Amostras contaminadas com sangue não foram incluídas neste estudo. Não foi feito lavado peritoneal. Pacientes que não apresentaram fluido peritoneal no momento 
da laparoscopia também não foram incluídas neste estudo.

Após a aspiração, o fluido peritoneal foi centrifugado a $2500 \mathrm{rpm}$ (1900 g) durante 15 minutos a $4^{\circ} \mathrm{C}$. Em seguida, o sobrenadante foi separado e estocado em tubos esterilizados a $-20^{\circ} \mathrm{C}$, até o momento das dosagens.

\section{Coleta de fluido folicular}

As amostras de fluido folicular foram obtidas por meio da punção de fundo de saco orientada pelo ultra-som transvaginal, com sedação prévia da paciente, 34 horas após a injeção de gonadotrofina coriônica humana. Neste estudo, foram utilizadas amostras de fluido folicular de folículos dominantes. Este critério permitiu a coleta de amostras mais homogêneas, livres de contaminação com sangue. Após a remoção do complexo oócito-cumulus oóforo, o fluido folicular aspirado foi imediatamente centrifugado a $1500 \mathrm{rpm}, \mathrm{a} 4^{\circ} \mathrm{C}$, durante 20 minutos. Em seguida, o sobrenadante livre de células foi separado e estocado em tubos esterilizados $\mathrm{a}-80^{\circ} \mathrm{C}$.

\section{Coleta de soro}

As amostras de sangue foram coletadas imediatamente antes da indução da anestesia, de pacientes submetidas à laparoscopia, realizada durante a fase secretora do ciclo menstrual. Não foi usado nenhum anticoagulante. O sobrenadante formado após a coagulação do sangue (soro) foi estocado em tubos a $-20^{\circ} \mathrm{C}$ até o momento das dosagens.

\section{Determinação das concentrações de FSH, $L H$, estradiol e progesterona}

As concentrações de FSH, LH, estradiol e progesterona foram determinadas com o Kit comercial da Diagnostics Products Corporation (DPC), Immulite System, Los Angeles, Califórnia, pelo método de quimioluminescência, realizado em duplicata.

Para o FSH, o coeficiente de variação intraensaio foi de $7,9,8,6$ e $10,2 \%$ no soro, no fluido peritoneal e no fluido folicular, respectivamente. $\mathrm{O}$ coeficiente de variação interensaio foi de 6,5, 9,2 e $8,1 \%$ no soro, no fluido peritoneal e no fluido folicular, respectivamente.

Para o LH, o coeficiente de variação intraensaio foi de 8,8, 7,6 e 9,4\% no soro, no fluido peritoneal e no fluido folicular, respectivamente. O coeficiente de variação interensaio foi de 11,3, 10,3 e $8,1 \%$ no soro, no fluido peritoneal e no fluido folicular, respectivamente.

Para o estradiol, o coeficiente de variação intraensaio foi de 8,4, 6,6 e 7,4\% no soro, no fluido peritoneal e no fluido folicular, respectivamente. $\mathrm{O}$ coeficiente de variação interensaio foi de $9,3,8,3$ e $8,2 \%$ no soro, no fluido peritoneal e no fluido folicular, respectivamente.

Para a progesterona, o coeficiente de variação intraensaio foi de $5,8,7,4$ e $8,4 \%$ no soro, no fluido peritoneal e no fluido folicular, respectivamente. $\mathrm{O}$ coeficiente de variação interensaio foi de 10,3, 11,5 e $8,9 \%$ no soro, no fluido peritoneal e no fluido folicular, respectivamente.

\section{Determinação da concentração de histamina}

Para a dosagem de histamina, amostras de fluido peritoneal e de fluido folicular foram adicionadas a $3 \mathrm{~mL}$ de ácido perclórico $0,8 \mathrm{~N}$. O conteúdo de histamina nas células foi liberado pela ruptura das células pelo ácido perclórico. A seguir, as amostras foram centrifugadas por 15 minutos a $2500 \mathrm{rpm}$, a $4^{\circ} \mathrm{C}$. Após a centrifugação, a porção orgânica foi removida e descartada. O sobrenadante foi coletado e estocado a $-20^{\circ} \mathrm{C}$ para avaliação da concentração de histamina.

O conteúdo de histamina foi determinado pelo método fluorimétrico descrito por Shore et al. ${ }^{12}$. A intensidade da fluorescência proporciona a base para o cálculo da concentração da histamina.

\section{Análise estatistica}

As concentrações de FSH, LH, estradiol, progesterona e histamina no soro, no fluido peritoneal e no fluido folicular dos diferentes subgrupos foram comparadas pelo teste de Kruskal-Wallis, complementado pelo teste de Dunn.

No fluido folicular, as concentrações de FSH, LH, estradiol, progesterona e histamina do grupo de mulheres com endometriose foram comparadas com o grupo de mulheres sem endometriose pelo teste $t$ de Student. A significância estatística foi definida como $\mathrm{p}<0,05$. Os resultados são apresentados como média \pm desvio padrão da média (SD).

\section{Resultados}

As concentrações de FSH e LH em mulheres com e sem endometriose estão apresentadas nas Tabelas 1 e 3 . Tanto no soro como no fluido peritoneal e no fluido folicular não houve diferença significativa entre mulheres com e sem endometriose. Os valores (média \pm desvio padrão) referentes às concentrações de FSH (mUI/ $\mathrm{mL}$ ) no soro foram: 4,11 $\pm 0,4$ para mulheres férteis sem endometriose; $3,5 \pm 0,3$ para mulheres inférteis com endometriose I-II e 4,5 $\pm 0,4$ para mulheres inférteis com endometriose III-IV. Os valores referentes às concentrações de FSH (mUI/mL) no fluido peritoneal foram: 
2,3 $\pm 0,2$ para mulheres férteis sem endometriose; $1,6 \pm 0,1$ para mulheres inférteis com endometriose I-II e $1,7 \pm 0,1$ para mulheres inférteis com endometriose III-IV. Os valores referentes às concentrações de $\mathrm{LH}(\mathrm{mUI} / \mathrm{mL})$ no soro foram: 2,9 $\pm 0,3$ para mulheres férteis sem endometriose; $2,7 \pm 0,3$ para mulheres inférteis com endometriose I-II e $3,9 \pm 0,3$ para mulheres inférteis com endometriose III-IV. Os valores referentes às concentrações de $\mathrm{LH}$ (mUI/mL) no fluido peritoneal foram: 1,7 $\pm 0,2$ para mulheres férteis sem endometriose; $1,1 \pm 0,1$ para mulheres inférteis com endometriose I-II e $1,5 \pm 0,1$ para mulheres inférteis com endometrio- se III-IV. Os valores referentes às concentrações de FSH e LH (mUI/mL) no fluido folicular foram, respectivamente: $6,3 \pm 0,8$ e $2,3 \pm 0,2$ para mulheres

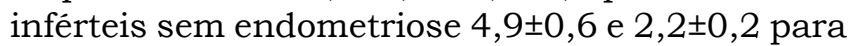
mulheres inférteis com endometriose.

As concentrações de estradiol e de progesterona em mulheres com e sem endometriose estão apresentadas nas Tabelas 2 e 3. No fluido peritoneal, as concentrações de estradiol $(\mathrm{E})$ e progesterona $(\mathrm{P})$ foram significativamente mais baixas em mulheres inférteis com endometriose (E: 154,2 $\pm 15,3$ para estágios I-II e $89,3 \pm 9,8 \mathrm{ng} / \mathrm{mL}$ para estágios III-IV; P:

Tabela 1 - Concentraç̃es de FSH e LH no soro e no fluido peritoneal (FP) em mulheres férteis sem endometriose e em mulheres inférteis com endometriose mínima-leve (endometriose I-II) e mulheres inférteis com endometriose moderada-severa (endometriose III-IV).

\begin{tabular}{|c|c|c|c|}
\hline Número total de pacientes $=49$ & $\begin{array}{c}\text { Mulheres férteis sem } \\
\text { endometriose } \\
(n=21)\end{array}$ & $\begin{array}{c}\text { Mulheres inférteis com } \\
\text { endometriose I-II } \\
(n=18)\end{array}$ & $\begin{array}{l}\text { Mulheres inférteis com } \\
\text { endometriose III-IV } \\
(n=10)\end{array}$ \\
\hline \multicolumn{4}{|l|}{ Parâmetros analisados } \\
\hline \multicolumn{4}{|l|}{$(\mathrm{mUl} / \mathrm{mL})$} \\
\hline FSH no soro & $4,1 \pm 0,4$ & $3,5 \pm 0,3$ & $4,5 \pm 0,4$ \\
\hline FSH no FP & $2,3 \pm 0,2$ & $1,6 \pm 0,1$ & $1,7 \pm 0,1$ \\
\hline LH no soro & $2,9 \pm 0,3$ & $2,7 \pm 0,3$ & $3,9 \pm 0,3$ \\
\hline LH no FP & $1,7 \pm 0,2$ & $1,1 \pm 0,1$ & $1,5 \pm 0,1$ \\
\hline
\end{tabular}

Os dados estão apresentados como média e desvio padrão da média. Não houve diferença significativa entre os grupos ( $\mathrm{p}<0,05$, teste de Kruskal-Wallis complementado pelo teste de Dunn).

Tabela 2 - Concentrações de estradiol, progesterona e histamina no soro e no fluido peritoneal $(F P)$ de mulheres férteis sem endometriose e mulheres inférteis com endometriose mínima-leve (endometriose I-II) e mulheres inférteis com endometriose moderada-severa (endometriose III-IV).

\begin{tabular}{|c|c|c|c|}
\hline Número total de pacientes $=49$ & $\begin{array}{c}\text { Mulheres férteis sem } \\
\text { endometriose } \\
(n=21)\end{array}$ & $\begin{array}{c}\text { Mulheres inférteis com } \\
\text { endometriose I-II } \\
(n=18)\end{array}$ & $\begin{array}{l}\text { Mulheres inférteis com } \\
\text { endometriose III-IV } \\
(n=10)\end{array}$ \\
\hline \multicolumn{4}{|l|}{$\begin{array}{l}\text { Parâmetros analisados } \\
\text { (pg/mL) }\end{array}$} \\
\hline Estradiol no soro & $100,5 \pm 12,4^{\mathrm{a}}$ & $77,5 \pm 10,3^{\text {ab }}$ & $56,7 \pm 6,2^{b}$ \\
\hline Estradiol no FP & $289,1 \pm 30,1^{\text {a }}$ & $154,2 \pm 15,3^{b}$ & $89,3 \pm 9,8^{c}$ \\
\hline Progesterona no soro & $9,8 \pm 0,8^{a}$ & $5,5 \pm 0,7^{b}$ & $3,8 \pm 0,4^{b}$ \\
\hline Progesterona no FP & $32,8 \pm 4,1^{a}$ & $11,2 \pm 1,5^{b}$ & $7,6 \pm 0,8^{b}$ \\
\hline Histamina no FP & $29,4 \pm 2,7^{\mathrm{a}}$ & $17,5 \pm 2,2^{b}$ & $15,4 \pm 1,1^{\mathrm{ab}}$ \\
\hline
\end{tabular}

Os dados estão apresentados como média e desvio padrão da média. Letras diferentes indicam diferença significativa entre os grupos, ( $p<0,05$, teste de Kruskal-Wallis complementado pelo teste de Dunn).

Tabela 3 - Concentrações de FSH, LH, estradiol, progesterona e histamina no fluido folicular de mulheres inférteis com e sem endometriose.

\begin{tabular}{|c|c|c|}
\hline Número total de pacientes $=39$ & $\begin{array}{l}\text { Mulheres inférteis sem endometriose } \\
\qquad(n=18)\end{array}$ & $\begin{array}{l}\text { Mulheres inférteis com endometriose } \\
\qquad(\mathrm{n}=21)\end{array}$ \\
\hline \multicolumn{3}{|l|}{ Parâmetros analisados } \\
\hline $\mathrm{FSH}(\mathrm{mUI} / \mathrm{mL})$ & $6,3 \pm 0,8$ & $4,9 \pm 0,6$ \\
\hline $\mathrm{LH}(\mathrm{mUl} / \mathrm{mL})$ & $2,3 \pm 0,2$ & $2,2 \pm 0,2$ \\
\hline Estradiol (pg/mL) & $237,5 \pm 28,5^{\mathrm{a}}$ & $97,4 \pm 11,1^{\mathrm{b}}$ \\
\hline Progesterona (pg/mL) & $558,2 \pm 70,2$ & $456,5 \pm 50,2$ \\
\hline Histamina (ng/mL) & $13,8 \pm 1,3^{a}$ & $6,6 \pm 0,9^{b}$ \\
\hline
\end{tabular}

Os dados estão apresentados como média e desvio padrão da média. Letras diferentes indicam diferença significativa entre os grupos. A ausência de letras indica que não houve diferença significativa entre os grupos ( $p<0.05$, teste $\boldsymbol{t}$ de Student). 
$11,2 \pm 1,5$ para estágios I-II e $7,6 \pm 0,8 \mathrm{ng} / \mathrm{mL}$ para estágios III-IV) em comparação com mulheres férteis sem endometriose $(\mathrm{E}: 289,1 \pm 30$, 1 ; P: $32,8 \pm 4,1 \mathrm{ng} / \mathrm{mL}$ ). No soro, as concentrações de estradiol e progesterona seguiram o mesmo padrão (E: $77,5 \pm 10,3$ para estágios I-II e $56,7 \pm 6,2 \mathrm{ng} / \mathrm{mL}$ para estágios III-IV; P: 5,5 $\pm 0,7$ para estágios I-II e $3,8 \pm 0,4 \mathrm{ng} / \mathrm{mL}$ para estágios III-IV) em comparação com mulheres férteis sem endometriose (E: $100,5 \pm 12,4 ; \mathrm{P}: 9,8 \pm 0,8 \mathrm{ng} / \mathrm{mL}$ )

No fluido folicular, as concentrações de estradiol (E) foram significativamente mais baixas em mulheres inférteis com endometriose (E: $97,4 \pm 11,1 \mathrm{pg} / \mathrm{mL}$ ) em comparação com mulheres inférteis sem endometriose (E: 237,5 $28,5 \mathrm{pg} / \mathrm{mL}$ ), enquanto os níveis de progesterona não revelaram diferença significativa entre os grupos (P: 558,21 $\pm 70,2 \mathrm{pg} / \mathrm{mL}$ em mulheres inférteis sem endometriose e 456,5 $\pm 50,2 \mathrm{pg} / \mathrm{mL}$ em mulheres inférteis com endometriose).

As concentrações de histamina (Hi) de mulheres com e sem endometriose estão apresentadas nas Tabelas 2 e 3

Tanto no fluido peritoneal (HiFP) como no fluido folicular (HiFF), as concentrações de histamina foram significativamente mais baixas em mulheres com endometriose (HiFP: 17,5 $\pm 2,2$ para estágios I-II e $15,4 \pm 1,1 \mathrm{ng} / \mathrm{mL}$ para estádios III-IV; HiFF: 6,6 $\pm 0,9$ ) em comparação com mulheres sem endometriose

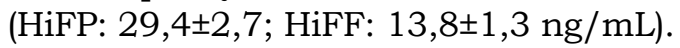

\section{Discussão}

Este estudo foi realizado para avaliar a associação entre a endometriose e as concentrações de fatores hormonais envolvidos no controle da função reprodutiva. A disfunção hipófise-ovário parece estar associada à endometriose em mulheres inférteis ${ }^{6,13}$. Estudos anteriores demonstraram que as concentrações séricas de $\mathrm{LH}^{14-16}$ e $\mathrm{FSH}^{15,16}$ não foram significativamente diferentes entre mulheres com e sem endometriose. Em contraste, uma redução significativa das concentrações plasmáticas de FSH em mulheres com endometriose foi relatada ${ }^{14}$.

No fluido peritoneal, estudos anteriores mostraram que as concentrações de FSH em mulheres com endometriose não foram estatisticamente diferentes quando comparadas aos seus respectivos controles. Em contraste ao FSH, as concentrações de LH nas fases folicular e lútea foram significativamente maiores em mulheres com endometriose ${ }^{17}$.

Nossos resultados mostraram que no soro, no fluido peritoneal e no fluido folicular os valores de FSH e LH não diferiram significativamente entre mulheres com e sem endometriose. A análise de nossos resultados e os dados da literatura mostram que não há dados conclusivos sobre a função hipófise-ovário em pacientes com endometriose. Embora a endometriose possa estar associada a alterações de mecanismos neuroendócrinos que controlam os níveis de FSH e $\mathrm{LH}$, nossos resultados e os dados da literatura mostram que nem sempre a endometriose está associada com uma disfunção hipófise-ovário e, neste caso, a subfertilidade em pacientes com endometriose pode estar associada a outras anormalidades do sistema reprodutivo.

Com relação às concentrações de estradiol e progesterona, os dados da literatura também são conflitantes. Os estágios iniciais de endometriose podem estar associados a níveis plasmáticos aumentados de estradiol em comparação com mulheres sem a doença ${ }^{14}$. A ausência de diferenças significativas nos niveis séricos de progesterona entre pacientes com e sem endometriose também foi relatada ${ }^{18}$. Por outro lado, resultados opostos têm sido observados por outros autores, os quais verificaram que as concentrações séricas de estradiol e progesterona estão diminuídas em mulheres com endometriose pélvica. As concentrações de progesterona também estão reduzidas no fluido peritoneal de mulheres com endometriose ${ }^{19,20}$. Além disso, os autores verificaram que a diminuição das concentrações de estradiol e progesterona em mulheres com endometriose estava associada à infertilidade. Outros autores também observaram uma redução das concentrações séricas de estradiol em mulheres inférteis com endometriose, em comparação com mulheres férteis sem endometriose $^{15}$. O mesmo grupo observou que mulheres com endometriose apresentaram concentrações séricas mais baixas de estradiol e secreção diminuída de progesterona durante a fase lútea ${ }^{20}$. No presente estudo, observamos que mulheres inférteis com endometriose apresentaram uma redução significativa de ambos os hormônios no soro e no fluido peritoneal em comparação com mulheres férteis sem endometriose.

O fluido peritoneal representa uma mistura de fluido tubáreo e de fluidos de origem peritoneal e ovariana. Normalmente, este fluido dinâmico apresenta muitos constituintes acelulares, incluindo esteróides ovarianos, hormônios e prostaglandinas, e elementos celulares, tais como monócitos e células endometriais. As concentrações de esteróides ovarianos são mais altas no fluido peritoneal que no plasma, uma vez que fluidos de origem ovariana derivam principalmente de folículos em desenvolvimento e do corpo lúteo ${ }^{21}$. 
Está bem estabelecido que os estrógenos estimulam diretamente o crescimento e desenvolvimento do tecido endometriótico ${ }^{21}$. Além disso, macrófagos peritoneais expressam receptores para estradiol e progesterona e, em mulheres com endometriose, macrófagos peritoneais produzem maior quantidade de fator de crescimento de hepatócitos após tratamento com estradiol, em comparação com macrófagos de mulheres sem endometriose. Estradiol e fator de crescimento de hepatócitos, em combinação ou isoladamente, estimulam a proliferação do estroma endometrial e podem estar envolvidos no desenvolvimento da endometriose ${ }^{22}$. Por se tratar de um micro-ambiente com produtos específicos e hormônios em concentrações diferentes daquelas encontradas no plasma ou no soro, o fluido peritoneal deve desempenhar uma função crucial na patogênese da endometriose e na infertilidade associada à endometriose $\mathrm{e}^{21}$.

No fluido folicular, um aumento na concentração de progesterona associado à severidade da doença já foi relatado ${ }^{23}$. Por outro lado, no fluido peritoneal, outros autores não observaram uma diferença significativa nas concentrações de estradiol e progesterona ${ }^{24,25}$.

Estudos anteriores mostraram que as concentrações de estradiol no fluido folicular são significativamente menores em mulheres com endometriose em comparação com mulheres sem a doença ${ }^{15,26,27}$. Em nosso estudo, também observamos que pacientes inférteis com endometriose apresentaram uma redução significativa na concentração intrafolicular de estradiol, em comparação com mulheres inférteis sem endometriose. Não detectamos nenhuma variação significativa nas concentrações de progesterona no fluido folicular de mulheres com e sem endometriose. A diminuição das concentrações de estradiol e progesterona no fluido peritoneal e a concentração intrafolicular reduzida de estradiol em mulheres inférteis com endometriose podem refletir uma produção prejudicada de esteróides pelas células granulosas. A produção de estradiol pelo folículo pode servir como um marcador da maturidade e também da qualidade do oócito. Como as pacientes selecionadas para a coleta de fluido folicular estavam sendo submetidas à fertilização in vitro, é possivel que folículos de mulheres com endometriose sejam menos responsivos a tratamentos que induzem a ovulação. De fato, já foi relatado que células granulosas de folículos pré-ovulatórios de mulheres inférteis com endometriose são menos sensiveis ao hormônio luteinizante ${ }^{28}$. Uma redução significativa da atividade aromatase em foliculos de mulheres com endometriose poderia explicar a produção diminuída de esteróides pelo folículo ${ }^{27}$. Todos estes dados em conjunto evidenciam que folículos de mulheres com endometriose apresentam esteroidogênese severamente prejudicada. Obviamente, uma esteroidogênese alterada pode afetar a função do oócito, o que poderia explicar a disfunção ovulatória, a fertilização prejudicada e a implantação defeituosa, parâmetros freqüentemente associados à endometriose.

Com relação à histamina, nossos resultados mostraram que mulheres inférteis com endometriose apresentaram concentrações mais baixas de histamina tanto no fluido peritoneal como no fluido folicular. Não houve diferença significativa entre mulheres com endometriose nos estágios I-II e mulheres com endometriose nos estágios III-IV. A histamina pode afetar o funcionamento do ovário, por afetar o fluxo sanguíneo para o ovário, além de estimular a contratilidade ovariana, a ovulação e a secreção de progesterona in vitro ${ }^{8}$. Além disso, a histamina também está envolvida na regulação da contratilidade uterina e na implantação do blastocisto ${ }^{9,29}$. Assim, é possível que alterações da concentração de histamina tanto no fluido peritoneal como no fluido folicular, possam afetar a foliculogênese, a ovulação, a concepção e a implantação do blastocisto. Deste modo, a redução da concentração da histamina, detectada em mulheres inférteis com endometriose, pode desempenhar um papel na deficiência reprodutiva associada à doença.

Em conclusão, neste estudo, observamos que mulheres com endometriose apresentaram concentrações reduzidas de estradiol e progesterona no soro e no fluido peritoneal. No fluido folicular, as concentrações de histamina e estradiol foram significativamente menores em mulheres com endometriose em comparação com mulheres sem endometriose. Nossos resultados e os dados da literatura apontam para uma provável disfunção ovulatória, com efeitos significativos nas concentrações de estradiol, progesterona e histamina, o que pode levar à subfertilidade freqüentemente associada à doença.

\section{Agradecimentos}

Agradecemos à Maria Albina Vercezi Bortolieiro por sua assistência técnica. 


\section{Referências}

1. Mahmood TA, Templeton A. Pathophysiology of mild endometriosis: review of literature. Hum Reprod. 1990;5(7):765-84.

2. Hull ML, Charnock-Jones DS, Chan CL, BrunerTran KL, Osteen KG, Tom BD, et al. Antiangiogenic agents are effective inhibitors of endometriosis. J Clin Endocrinol Metab. 2003;88(6):2889-99.

3. Siristatidis C, Nissotakis C, Chrelias C, Iacovidou H, Salamalekis E. Immunological factors and their role in the genesis and development of endometriosis. J Obstet Gynaecol Res. 2006;32(2):162-70.

4. Agic A, Xu H, Finas D, Banz C, Diedrich K, Hornung D. Is endometriose associated with systemic subclinical inflammation? Gynecol Obstet Invest. 2006;62(3):139-47.

5. Hill JA. Immunology and endometriosis. Fertil Steril. 1992;58(2):262-4.

6. Cahill DJ, Hull MG. Pituitary-ovarian dysfunction and endometriosis. Hum Reprod Update. 2000; 6(1):56-66.

7. Trinder J, Cahill DJ. Endometriosis and infertility: the debate continues. Hum Fertil (Camb). 2002;5(1 Suppl):S21-7.

8. Krishna A, Beesley K, Terranova PF. Histamine, mast cells and ovarian function. J Endocrinol. 1989;120(3):363-71.

9. Jones RE, Duvall D, Guillette LJ Jr, Lopez KH. Number and state of rat ovarian mast cells after exogenous administration of luteinizing hormone. Comp Biochem Physiol Comp Physiol. 1994;108(4):555-9.

10.Szelag A, Merwid-Lad A, Trocha M. Histamine receptors in the female reproductive system. Part I. Role of the mast cells and histamine in female reproductive system. Ginekol Pol. 2002;73(7):627-35.

11.Revised American Society for Reproductive Medicine classification of endometriosis: 1996. Fertil Steril. 1997;67(5):817-21.

12.Shore PA, Burkhalter A, Cohn VH Jr. A method for the flurometric assay of histamine in tissues. $J$ Pharmacol Exper Ther. 1959;127:182-6.

13.Bancroft K, Vaughan Williams CA, Elstein M. Pituitaryovarian function in women with minimal or mild endometriosis and otherwise unexplained infertility. Clin Endocrinol (Oxf). 1992;36(2):177-81.

14.Provinciali M, Di Stefano G, Muzzioli M, Garzetti GG, Ciavattini A, Fabris N. Relationship between 17-beta-estradiol and prolactin in the regulation of natural killer cell activity during progression of endometriosis. J Endocrinol Invest. 1995; 18(8):645-52.
15.Cunha-Filho JS, Gross JL, Lemos NA, Brandelli A, Castillos M, Passos EP. Hyperprolactinemia and luteal insufficiency in infertile patients with mild and minimal endometriosis. Horm Metab Res. 2001;33(4):216-20.

16.Omland AK, Fedorcsak P, Storeng R, Dale PO, Abyholm T, Tanbo T. Natural cycle IVF in unexplained, endometriosis-associated and tubal factor infertility. Hum Reprod. 2001;16(12):25 87-92.

17. Chew PC, Peh KL, Loganath A, Gunasegaram R, Ratnam SS. Elevated peritoneal fluid luteinizing hormone and prolactin concentrations in infertile women with endometriosis. Int J Gynaecol Obstet. 1990;33(1):35-9.

18.Tizingounis V, Maghioracos P. Luteal insufficiency and endometriosis. Comparative study of plasma progesterone and of the evolutionary stage. J Gynecol Obstet Biol Reprod (Paris). 1985;14(4): 455-8.

19.Kusuhara K. Luteal function in infertile patients with endometriosis. Am J Obstet Gynecol. 1992;167(1): 274-7.

20.Cunha-Filho JS, Gross JL, Bastos de Souza CA, Lemos NA, Giugliani C, Freitas F, et al. Physiopathological aspects of corpus luteum defect in infertile patients with mild/minimal endometriosis. J Assist Reprod Genet. 2003;20(3):117-21.

21.Koninckx PR, Kennedy SH, Barlow DH. Pathogenesis of endometriosis: the role of peritoneal fluid. Gynecol Obstet Invest. 1999;47 Suppl 1:23-33.

22.Khan KN, Masuzaki H, Fujishita A, Kitajima M, Sekine I, Matsuyama T, et al. Estrogen and progesterone receptor expression in macrophages and regulation of hepatocyte growth factor by ovarian steroids in women with endometriosis. Hum Reprod. 2005;20(7):2004-13.

23.Pellicer A, Albert C, Mercader A, Bonilla-Musoles F, Remohi J, Simon C. The follicular and endocrine environment in women with endometriosis: local and systemic cytokine production. Fertil Steril. 1998;70(3):425-31.

24.De Leon FD, Vijayakumar R, Brown M, Rao CV, Yussman MA, Schultz G. Peritoneal fluid volume, strogen, progesterone, prostaglandin, and epidermal growth factor concentrations in patients with and without endometriosis. Obstet Gynecol. 1986;68(2):189-94.

25.Mahmood TA, Templeton A. Peritoneal fluid volume and sex steroids in the pre-ovulatory period in mild endometriosis. Br J Obstet Gynaecol. 1991;98(2):179-83.

26.Wunder DM, Mueller MD, Birkhauser $\mathrm{MH}$, Bersinger NA. Steroids and protein markers in the 
follicular fluid as indicators of oocyte quality in patients with and without endometriosis. J Assist Reprod Genet. 2005;22(6):257-64.

27. Harlow CR, Cahill DJ, Maile LA, Talbot WM, Mears $\mathrm{J}$, Wardle PG, et al. Reduced preovulatory granulosa cell steroidogenesis in women with endometriosis. J Clin Endocrinol Metab. 1996;81(1):426-9.
28.Cahill DJ, Harlow CR, Wardle PG. Pre-ovulatory granulosa cells of infertile women with endometriosis are less sensitive to luteinizing hormone. Am J Reprod Immunol. 2003;49(2):66-9.

29.Parshad RK, Kathpalia K. Distribution and characteristics of mast cells in immature rat ovary. Indian J Exp Biol. 1988;26(12):937-40. 